To the Editors:

\title{
Central vascular catheter-related blood stream infections: incidence, associated pathogens and antibiotic sensitivity patterns at a tertiary care hospital in Sri Lanka
}

Central vascular catheter related blood stream infections (CRBSIs) are defined as presence of bacteraemia originating from an intravascular device. CRBSIs cause a substantial amount of morbidity, mortality and increased hospital stay.

From February to November 2006, 100 central venous catheters (CVCs) were collected from patients admitted consecutively to the medical and surgical intensive care units (ICUs) of Colombo South Teaching Hospital (CSTH), who had temperature $>38^{\circ} \mathrm{C}$. Before removal of the venous catheter, two blood culture samples were collected, one through the central venous line and the other from a peripheral vein. Ethical committee approval and informed consent were obtained before collecting samples. Catheter tips were processed according to a quantitative technique known as the sonication method. The distal $4 \mathrm{~cm}$ of the catheter tip was placed in brain heart infusion broth followed by sonication and then vortexing. The number of colony forming units (cfu) was determined by serial dilution. The threshold for a significant count was $\geq 10^{3}$ $\mathrm{cfu} / \mathrm{ml}$. Blood cultures were processed according to guidelines issued by the College of Microbiologists of Sri Lanka. The organisms were then identified by routine microbiological techniques using API strips.

CRBSI was confirmed in $11 \%$. Catheter colonization without bacteraemia was seen in $16 \%$ and there was no growth in $70 \%$ of catheters. In one sample $(1 \%)$, the tip culture was negative while both blood cultures grew the same organism. Two (2\%) samples grew different organisms from the catheter tip and peripheral blood cultures, representing bacteraemia not associated with intravascular catheters. Various rates of CRBSIs have been reported from studies done in many countries, ranging from $2 \%$ to $34 \%[1,2,3]$. The rate of CRBSIs $(11 \%)$ in the present study lies in between this range.

Pathogens responsible for CRBSIs in present study were Klebsiella pneumoniae and Candida species which were isolated in two patients each and coagulase-negative staphylococci, Acinetobacter baumannii/calcoaceticus, Enterobacter cloacae, Brevundimonas vesicularis, Burkholderia cepacia, Chryseomonas luteola and Enterococcus species in one patient each. Therefore, the majority of aetiological agents were Gram-negative bacteria (64\%), while Gram-positive bacteria and yeasts accounted for $18 \%$ each.

Studies have shown coagulase-negative Staphylococci as the predominant aetiological agent of CRBSI [4,5]. Use of povidone iodine for skin disinfection may be a reason for the rarity of Gram-positive organisms in our study.

Most of these organisms were resistant to the first line antibiotics and to some of the second line antibiotics. The isolate A. baumannii/calcoaceticus was a panresistant organism. The emergence of multi resistant pathogens in ICUs is high, due to the heavy usage of antibiotics for critically ill patients. This highlights a high rate of antibiotic resistance among hospital pathogens in Sri Lanka.

\section{Acknowledgement}

We thank IRQUE for funding the IRQUE/CBR/RG/ 2006/31 grant.

\section{References}

1. Charalambos C, James D, Pamela A, Sandra MS, Trish P. Risk factors and clinical impact of central line infections in the Surgical Intensive Care Unit. Archives of Surgery 1998; 133: $1241-6$.

2. Crossley K, Matsen JM. Intravenous catheter-associated bacteremia: role of the diagnostic microbiology laboratory. Applied Microbiology 1973; 26: 1006-7.

3. Geraldo S, Filho AD, Filho PPG. Central venous catheterrelated bloodstream infection caused by Staphylococcus aureus: microbiology and risk factors. Brazilian Journal of Infectious Diseases 2006; 10: 1413-6.

4. Richet H, Hubert B, Nitemberg G. et al. Prospective multicenter study of vascular-catheter-related complications and risk factors for positive central-catheter cultures in intensive care unit patients. Journal of Clinical Microbiology 1990; 28: 2520-5.

5. Leonardo L, Henry C, Martín MM, Jiménez A, Mora ML. Central venous catheter-related infection in a prospective and observational study of 2,595 catheters. Critical Care 2005; 9: 631-5.

\footnotetext{
K D K P Kumari ${ }^{1}$, R Rajapakse Mallikahewa ${ }^{1}$, S Chandasiri², M Nanayakkara ${ }^{2}$, D F D Meedin ${ }^{1}$ and S S N Fernando ${ }^{1}$ ${ }^{1}$ Department of Microbiology, Faculty of Medical Sciences, University of Sri Jayewardenepura, Sri Lanka.

${ }^{2}$ Microbiology Unit, Colombo South Teaching Hospital, Sri Lanka.

Correspondence: NF, e-mail <fernando_neluka@Yahoo.com>. Received 18 February 2009 and revised version accepted 1 October 2009. Competing interests: none declared.
} 